\title{
SUPPLEMENT TO: GOODNESS OF FIT TESTS FOR A CLASS OF MARKOV RANDOM FIELD MODELS
}

\author{
By Mark S. Kaiser, Soumendra N. Lahiri And \\ DANIEL J. NORDMAN \\ Iowa State University and Texas A\&M University
}

This supplement provides proofs of all asymptotic distributional results from Section 4 of the main manuscript, regarding the conclique-based spatial goodness of fit (GOF) test statistics in null and composite hypothesis settings (Proposition 4.1, Theorem 4.2, Corollary 4.3, Theorem 4.4, Corollary 4.5). In what follows, these proofs are divided into subsections of an Appendix A, and depend on assumptions/conditions described in Section 4 of the main manuscript.

Appendix A.1 concerns the proof of the "basic conclique" $\mathcal{C}_{j}^{*}$ properties in Proposition 4.1, where the collection of disjoint sublattices defined as

$$
\mathcal{C}_{j}^{*}=\left\{\mathbf{a}_{j}+\Delta \mathbf{s}: \mathbf{s} \in \mathbb{Z}^{d}\right\}, \quad j=1, \ldots, q^{*} \equiv \prod_{i=1}^{d}\left(m_{i}+1\right),
$$

with $\mathbf{a}_{j} \neq \mathbf{a}_{k}$ if $\mathcal{C}_{j}^{*} \neq \mathcal{C}_{k}^{*}$ for

$$
\mathbf{a}_{j} \in \mathcal{I} \equiv\left\{\left(a_{1}, \ldots, a_{d}\right)^{\prime} \in \mathbb{Z}^{d}: 0 \leq a_{i} \leq m_{i}, i=1, \ldots, d\right\}
$$

can be generally used to build a collection of concliques $\mathcal{C}_{1}, \ldots, C_{q}$ on the regular lattice $\mathbb{Z}^{d}$; see equation (4.1) of the main manuscript and Condition (C.1). (Recall above $\Delta=\operatorname{diag}\left(m_{1}+1, \ldots, m_{d}+1\right.$ ) is a positive diagonal matrix with $m_{i} \equiv \max \left\{\left|\mathbf{e}_{i}^{\prime} \mathbf{s}\right|: \mathbf{s} \in \mathcal{M}\right\}, i=1, \ldots, d$, as the maximal absolute value of $i$ th component over integer vectors in the neighborhood template $\mathbf{s} \in \mathcal{M} \subset \mathbb{Z}^{d} \backslash\{\mathbf{0}\}(|\mathcal{M}|<\infty)$, and $\mathbf{e}_{i} \in \mathbb{Z}^{d}$ denotes a standard coordinate vector with 1 in the $i$ th component.)

Appendix A.2 then addresses proofs in the simple null hypothesis case (Theorem 4.2, Corollary 4.3), while Appendix A.3 provides (non-standard) proofs in the composite null hypothesis case (Theorem 4.4 and Corollary 4.5). An Appendix B collects the proofs of two further technical results required in Appendix A.3. All literature citations in the following appear in a Reference Section at the end of this supplement. 
To ease notation, for $A \subset \mathbb{Z}^{d}$, we write $\mathcal{Y}(A) \equiv\{Y(\mathbf{s}): \mathbf{s} \in A\}$ in the following and let $\mathcal{C}_{k n}^{*}=\mathcal{C}_{k}^{*} \cap \mathcal{S}_{N_{n}}^{\text {int }}, 1 \leq k \leq q^{*}$, and $\mathcal{C}_{j n}=\cup_{k \in \mathcal{J}_{j}} \mathcal{C}_{k n}^{*}$, $1 \leq j \leq q$ under Condition (C.1). Also, let $C, C(\cdot) \in(0, \infty)$ denote generic constants that depend on their arguments, but not on $n$. Let $\lfloor x\rfloor$ denote the integer part of a real number $x$. For a vector $\mathbf{x}=\left(x_{1}, \ldots, x_{k}\right)^{\prime}$, we write $\|\mathbf{x}\|=\left(x_{1}^{2}+\ldots+x_{k}^{2}\right)^{1 / 2}$.

\section{APPENDIX A: PROOFS OF MAIN RESULTS}

A.1. Conclique Properties in Proposition 4.1. Let $\mathcal{C}_{j}^{*}, \mathrm{a}_{j} \in \mathcal{I}$, denote the sublattice collection from above, corresponding to equation (4.1) of Section 4 . Then, for any two points $\mathbf{s} \neq \mathbf{t} \in \mathcal{C}_{j}^{*}$, the $\|\cdot\|_{\infty}$-distance between $\mathbf{s}$ and $\mathcal{N}(\mathbf{t})=\mathbf{t}+\mathcal{M}$ is bounded below by the distance

$$
\begin{aligned}
d_{\infty}(\mathbf{s}, \mathbf{t}+\mathcal{M}) & \equiv \min \left\{\|\mathbf{s}-\mathbf{t}-\mathbf{y}\|_{\infty}: \mathbf{y} \in \mathcal{M}\right\} \\
& \geq \max \left\{\left|\mathbf{e}_{j}^{\prime}(\mathbf{s}-\mathbf{t})\right|-\max \left\{\left|\mathbf{e}_{j}^{\prime} \mathbf{y}\right|: \mathbf{y} \in \mathcal{M}\right\}: j=1, \ldots, d\right\} \\
& \geq 1,
\end{aligned}
$$

implying that $\mathbf{s}$ is not a neighbor of $\mathbf{t}$. By similar arguments, $\mathbf{t}$ is not a neighbor of $\mathbf{s}$, implying $\mathcal{C}_{j}^{*}$ is a conclique and establishing part (a).

If $\mathbf{a}_{1} \neq \mathbf{a}_{2} \in \mathcal{I}$, then $\mathcal{C}_{1}^{*} \cup \mathcal{C}_{2}^{*}$ is a conclique if and only if $\mathbf{t}_{1}$ is not a neighbor of $\mathbf{t}_{2}$ (i.e., $\min _{i=1,2} d_{\infty}\left(\mathbf{t}_{i}, \mathbf{t}_{3-i}+\mathcal{M}\right) \geq 1$ ) for any $\mathbf{t}_{1} \in \mathcal{C}_{1}^{*}, \mathbf{t}_{2} \in \mathcal{C}_{2}^{*}$ which is equivalent to $\min _{i=1,2} d_{\infty}\left(\mathbf{a}_{i}-\mathbf{a}_{3-i}+\Delta \mathbf{s}, \mathcal{M}\right) \geq 1$ for any $\mathbf{s} \in \mathbb{Z}^{d}$; the latter always holds for $\|\mathbf{s}\|>1$ by an argument as in part (a). Hence, $\mathcal{C}_{1}^{*} \cup \mathcal{C}_{2}^{*}$ is a conclique if and only if $\mathbf{a}_{1}-\mathbf{a}_{2}+\Delta \mathbf{s} \notin \pm \mathcal{M}$ holds for all $\mathbf{s} \in \mathbb{Z}^{d}$, $\|\mathbf{s}\|_{\infty} \leq 1$. Part(b) now follows iteratively.

A.2. Proofs of Results in Simple Null Case. We focus on establishing Theorem 4.2, and Corollary 4.3 will then follow this and the continuous mapping theorem for weak convergence (cf. Theorem 1.9.5, [3]).

Let $\mathbf{W}_{n}^{*}=\left(W_{1 n}^{*}, \ldots, W_{q^{*} n}\right)^{\prime}$ denote the empirical process version of $\mathbf{W}_{n}=$ $\left(W_{1 n}, \ldots, W_{q n}\right)^{\prime}$ (see Section 4.3 and Condition (C.1)) defined with respect to basic concliques in (4.1) so that

$$
W_{j n}^{*}(u)=\frac{N_{n}^{1 / 2}}{\left|\mathcal{C}_{j n}^{*}\right|} \sum_{\mathbf{s} \in \mathcal{C}_{j n}^{*}}[\mathbb{I}(U(\mathbf{s}) \leq u)-u], u \in[0,1],
$$

$j=1, \ldots, q^{*} \equiv \operatorname{det}(\Delta)$ and $W_{j n}(u)=\sum_{k \in \mathcal{J}_{j}}\left|\mathcal{C}_{k n}^{*}\right| W_{k n}^{*}(u) /\left|\mathcal{C}_{j n}\right|, j=1, \ldots, q$, under condition (C.1). Let $\mathbf{W}^{*}(u)=\left(W_{1}^{*}(u), \ldots, W_{q^{*}}^{*}(u)\right), u \in[0,1]$, be a 
mean-zero Gaussian process (continuous sample paths on $[0,1]$ with probability 1 and $P\left(\mathbf{W}^{*}(u)=\mathbf{0}\right)=1$ for $\left.u=0,1\right)$ and covariances given by

$$
E W_{j}^{*}(u) W_{k}^{*}(v)= \begin{cases}\operatorname{det}(\Delta)(\min \{u, v\}-u v) & \text { if } j=k \\ \operatorname{det}(\Delta) \sigma_{j, k}(u, v) & \text { if } j \neq k\end{cases}
$$

for $0 \leq u, v \leq 1,1 \leq j, k \leq q^{*}$. Then, $\mathbf{W}_{n}^{*} \stackrel{d}{\rightarrow} \mathbf{W}^{*}$ holds as $n \rightarrow \infty$. (Because the generalized residuals are $m$-dependent under Proposition 4.1 , weak convergence of the finite dimensional distributions of $\mathbf{W}_{n}^{*}(\cdot)$ follows from the central limit theorem result [1] and Cramer-Wold device. In view of Theorem 2.1 on the distribution of the generalized residuals from a given conclique, tightness of the component processes $W_{j n}^{*}(\cdot)$ (and hence of $\mathbf{W}_{n}^{*}$ ) follows from standard results on weak convergence of the iid Uniform $(0,1)$-random variables.) Since $\left|\mathcal{C}_{k n}^{*}\right| /\left|\mathcal{C}_{j n}\right| \rightarrow 1 /\left|\mathcal{J}_{j}\right|$ as $n \rightarrow \infty$ for $k \in \mathcal{J}_{j}, j=1, \ldots, q$, it then follows that $\mathbf{W}_{n} \stackrel{d}{\rightarrow} \mathbf{W}$, proving Theorem 4.2.

A.3. Proofs of Results in Composite Null Case. This section is devoted to proving Theorem 4.4. For the ease of reference later on, here we collect the necessary notation. Let $\mathbf{Z}_{n}=N_{n}^{1 / 2}\left(\hat{\theta}_{n}-\theta_{0}\right)$. For $\mathbf{s} \in \mathbb{Z}^{d}$ and $\theta_{0}+\mathbf{x} \in \Theta$, define $\tilde{U}(\mathbf{s}, \mathbf{x})=(1-A(\mathbf{s})) \cdot F_{\theta_{0}+\mathbf{x}}(Y(\mathbf{s}) \mid \mathcal{Y}(\mathcal{N}(\mathbf{s})))+A(\mathbf{s})$. $F_{\theta_{0}+\mathbf{x}}^{-}(Y(\mathbf{s}) \mid \mathcal{Y}(\mathcal{N}(\mathbf{s})))$. In the main manuscript, note that, in $(3.2), U(\mathbf{s})=$ $\tilde{U}(\mathbf{s}, \mathbf{0})$ and, in $(3.8), \hat{U}_{n}(\mathbf{s})=\tilde{U}\left(\mathbf{s}, \mathbf{Z}_{n} / N_{n}^{1 / 2}\right), \mathbf{s} \in \mathbb{Z}^{d}$. For $j=1, \ldots, q$, define $\xi_{j n}(\mathbf{b}, u)=N_{n}^{1 / 2}\left[\left|\mathcal{C}_{j n}\right|^{-1} \sum_{\mathbf{s} \in \mathcal{C}_{j n}} \mathbb{I}\left(\tilde{U}\left(\mathbf{s}, \mathbf{b} / N_{n}^{1 / 2}\right) \leq u\right)-u\right] \mathbb{I}\left(\mathbf{b} \in B_{n}\right)$, $\check{\xi}_{j n}(\mathbf{b}, u)=N_{n}^{1 / 2}\left[\left|\mathcal{C}_{j n}\right|^{-1} \sum_{\mathbf{s} \in \mathcal{C}_{j n}} \mathbb{I}\left(\tilde{U}\left(\mathbf{s}, \mathbf{b} / N_{n}^{1 / 2}\right) \leq u\right)-H_{n}(\mathbf{b}, u)\right] \mathbb{I}\left(\mathbf{b} \in B_{n}\right)$,

for all $u \in[0,1]$ and for all $\mathbf{b} \in \mathbb{R}^{p}$, where $B_{n} \equiv\left\{\mathbf{x}: \theta_{0}+\mathbf{x} / N_{n}^{1 / 2} \in \Theta\right\}$ and where

$$
H_{n}(\mathbf{b}, u)=P\left(\tilde{U}\left(\mathbf{s}, \mathbf{b} / N_{n}^{1 / 2}\right) \leq u\right) .
$$

Then, recalling the conclique-wise empirical processes $\widehat{\mathbf{W}}_{n}=\left(\widehat{W}_{1 n}, \ldots, \widehat{W}_{q n}\right)^{\prime}$ based on scaled and centered empirical distributions $\widehat{W}_{j n}(u), u \in[0,1]$, from (3.9), it follows that

$$
\begin{aligned}
\widehat{W}_{j n}(u) & =\xi_{j n}\left(\mathbf{Z}_{n}, u\right), \\
& =\check{\xi}_{j n}\left(\mathbf{Z}_{n}, u\right)+N_{n}^{1 / 2}\left[H_{n}\left(\mathbf{Z}_{n}, u\right)-u\right] \\
& \equiv h_{j n}\left(\check{\xi}_{n}, \mathbf{Z}_{n}\right), \text { say }
\end{aligned}
$$


$u \in[0,1], j=1, \ldots, q$, where $\check{\xi}_{n}(\mathbf{b}, u)=\left(\check{\xi}_{1 n}(\mathbf{b}, u), \ldots, \check{\xi}_{q n}(\mathbf{b}, u)\right)^{\prime}$ is a $q-$ vector process indexed by $\mathbf{b} \in \mathbb{R}^{p}$ and $u \in[0,1]$. Write $\mathbf{h}_{n}=\left(h_{1 n}, \ldots, h_{q n}\right)^{\prime}$. Then,

$$
\widehat{\mathbf{W}}_{n}=\mathbf{h}_{n}\left(\check{\xi}_{n}, \mathbf{Z}_{n}\right) .
$$

First we will establish the limit distribution of $\left(\check{\xi}_{n}, \mathbf{Z}_{n}\right)$ and then use the extended continuous mapping theorem to derive the limit distribution of $\widehat{\mathbf{W}}_{n}$. We need the following lemma to prove tightness of the process $\left(\check{\xi}_{n}, \mathbf{Z}_{n}\right)$; the proof is deferred to the technical Appendix B.1.

Lemma A.1: For each $n \geq 1$, let $\left\{\varepsilon_{n}(\mathbf{i}): \mathbf{i} \in \mathbb{Z}^{d}\right\}$ be a collection of Bernoulli random variables satisfying $P\left(\varepsilon_{n}(\mathbf{i})=1\right)=\pi_{n}(\mathbf{i})=1-P\left(\varepsilon_{n}(\mathbf{i})=0\right), \mathbf{i} \in \mathbb{Z}^{d}$ for some $\pi_{n}(\mathbf{i}) \in[0,1]$. Suppose that $\varepsilon_{n}(\mathbf{i}) \in \sigma\langle\mathcal{Y}(\mathcal{N}(\mathbf{i}))\rangle$ for all $\mathbf{i} \in \mathbb{Z}^{d}$ and $n \geq 1$, and that $\max \left\{\pi_{n}(\mathbf{i}): \mathbf{i} \in \mathcal{S}_{N_{n}}\right\} \leq \pi_{0 n}$ for some $0<\pi_{0 n} \leq 1$. Then, for any integer $r \geq 1$ and any real numbers $a>1$ and $b>2$ with $\frac{1}{a}+\frac{2}{b}=1$, there exists a constant $C=C\left(d, a, A_{r}\right) \in(0, \infty)$ such that

$$
E\left|\sum_{\mathbf{s} \in \mathcal{S}_{N_{n}}}\left[\varepsilon_{n}(\mathbf{s})-\pi_{n}(\mathbf{s})\right]\right|^{2 r} \leq C \sum_{j=1}^{r} N_{n}^{j}\left[\pi_{0 n}^{2 / b}\right]^{j}
$$

for all $n \geq 1$, where $A_{r}=\max \left\{1+\sum_{j=1}^{\infty} j^{d(m-1)} \alpha(j, m-1)^{1 / a}: 2 \leq m \leq 2 r\right\}$.

To then use the representation (A.2) of $\widehat{\mathbf{W}}_{n}$ and the extended continuous mapping theorem (cf. Theorem 1.11.1, [3]) to establish the limit distribution, we require some additional notation as defined follows:

$$
\begin{aligned}
\mathbb{D} & =\left(\mathcal{L}_{c}^{\infty}\left(\mathbb{R}^{p} \times[0,1]\right)\right)^{q} \times \mathbb{R}^{p}, \\
\mathbb{D}_{n} & =\left\{\mathbf{f} \in \mathbb{D}: \mathbf{f}(\mathbf{b}, u)=0 \text { for all }(\mathbf{b}, u) \notin B_{n} \times[0,1]\right\}, n \geq 1 \\
\mathcal{L}_{\infty}^{q} & =\left(\mathcal{L}^{\infty}([0,1])\right)^{q},
\end{aligned}
$$

where for any nonempty set $A, \mathcal{L}^{\infty}(A)$ denotes the space of all bounded functions from $A \rightarrow \mathbb{R}$ equipped with the sup-norm and where $\mathcal{L}_{c}^{\infty}\left(\mathbb{R}^{p} \times[0,1]\right)$ is the space of locally bounded functions from $\mathbb{R}^{p} \times[0,1]$ to $\mathbb{R}$, equipped with the norm

$$
\|f\|_{c}=\sum_{i=1}^{\infty} 2^{-i} \sup \left\{|f(\mathbf{b}, u)|:(\mathbf{b}, u) \in D_{i}\right\},
$$

$f \in \mathcal{L}_{c}^{\infty}\left(\mathbb{R}^{p} \times[0,1]\right)$, with $D_{i}=[-i, i]^{p} \times[0,1], i \geq 1$. For $f_{1} \in \mathcal{L}_{\infty}^{q}$, define the norm $\left\|f_{1}\right\|_{\mathcal{L}_{\infty}^{q}}=\sup \left\{\left\|f_{1}(u)\right\|: u \in[0,1]\right\}$. Let $\mathbb{D}_{0}=(\mathcal{C}[0,1])^{q} \times \mathbb{R}^{p}$, where 
$\mathcal{C}[0,1]$ is the space of continuous functions from $[0,1] \rightarrow \mathbb{R}$. We use the canonical embedding of $\mathbb{D}_{0}$ in $\mathbb{D}$ where an element $\left(\mathbf{f}_{0}, \mathbf{b}\right) \in \mathbb{D}_{0}$ is identified with $(\mathbf{f}, \mathbf{b}) \in \mathbb{D}$ where $\mathbf{f}(\mathbf{b}, u)=\mathbf{f}_{0}(u)$.

Note that $P\left(\left(\check{\xi}_{n}, \mathbf{Z}_{n}\right) \in \mathbb{D}_{n}\right)=1$ for all $n \geq 1$, and $P\left((\mathbf{W}, \mathbf{Z}) \in \mathbb{D}_{0}\right)=1$. By Theorem 1.11.1 of [3], it is now enough to show that

(i) $\left(\check{\xi}_{n}, \mathbf{Z}_{n}\right) \stackrel{d}{\rightarrow}(\mathbf{W}, \mathbf{Z})$ as random elements of $\mathbb{D}$,

(ii) $\mathbf{h}_{n}\left(y_{n}\right) \rightarrow \mathbf{h}(y)$ when $y_{n} \rightarrow y$ with $y_{n} \in \mathbb{D}_{n}, n \geq 1, y \in \mathbb{D}_{0}$,

where $\mathbf{h}: \mathbb{D}_{0} \rightarrow \mathcal{L}_{\infty}^{q}$ is defined by

$$
\mathbf{h}(\mathbf{w}, \mathbf{z})=\mathbf{w}+\mathbf{1} \cdot \mathbf{z}^{\prime} \int_{\mathbb{R}^{p}} \mathbf{x} \tilde{f}(\cdot, \mathbf{x}) \mu(d \mathbf{x}), \quad(\mathbf{w}, \mathbf{z}) \in \mathbb{D}_{0} .
$$

First consider (ii) of (A.3). Suppose that $y_{n}=\left(\mathbf{f}_{n}, \mathbf{b}_{n}\right) \in \mathbb{D}_{n}, n \geq 1$, and $y=\left(\mathbf{f}_{0}, \mathbf{b}\right) \in \mathbb{D}_{0}$ such that $y_{n} \rightarrow y$ in $\mathbb{D}$. For $M \in(0, \infty)$, define

$$
\begin{aligned}
& \Delta_{1 n}(M)= \sup \left\{\left\|\mathbf{f}_{n}(\mathbf{t}, u)-\mathbf{f}_{0}(u)\right\|:\|\mathbf{t}\| \leq M, u \in[0,1]\right\} \\
& \Delta_{2 n}(M)= \sup \left\{\left|N_{n}^{1 / 2}\left[H_{n}(\mathbf{b}, u)-u\right]-\mathbf{b}^{\prime} \int \mathbf{x} \tilde{f}(u, \mathbf{x}) \mu(d \mathbf{x})\right|:\right. \\
&u \in[0,1],\|\mathbf{b}\| \leq M\} .
\end{aligned}
$$

We then require the following result and defer its proof to the technical Appendix B.2.

Lemma A.2: Let Conditions (C.2)-(C.3) hold. Then, for every $M>0$, $\Delta_{2 n}(M) \rightarrow 0$ as $n \rightarrow \infty$.

Since $y_{n} \rightarrow y$ in $\mathbb{D}$, for every $M \in(0, \infty)$,

$$
\Delta_{1 n}(M)+\left\|\mathbf{b}_{n}-\mathbf{b}\right\| \rightarrow 0 \quad \text { as } n \rightarrow \infty
$$

Now assume $M_{0}=\sup \left\{\left\|\mathbf{b}_{n}\right\|: n \geq 1\right\}<\infty$ by (A.4). Using Lemma A.2 and (A.4), we have that (ii) of (A.3) follows from

$$
\begin{aligned}
& \left\|\mathbf{h}_{n}\left(y_{n}\right)-\mathbf{h}(y)\right\|_{\mathcal{L}_{\infty}^{q}} \\
\leq & \sup _{u \in[0,1]}\left\|\mathbf{f}_{n}\left(\mathbf{b}_{n}, u\right)-\mathbf{f}_{0}(u)\right\|+ \\
& \quad \sup _{u \in[0,1]}\left|N_{n}^{1 / 2}\left[H_{n}\left(\mathbf{b}_{n}, u\right)-u\right]-\mathbf{b}^{\prime} \int \mathbf{x} \tilde{f}(u, \mathbf{x}) \mu(d \mathbf{x})\right| \\
\leq & \Delta_{1 n}\left(M_{0}\right)+C \Delta_{2 n}\left(M_{0}\right)+\left\|\mathbf{b}_{n}-\mathbf{b}\right\| \sup _{u \in[0,1]}\left\|\int \mathbf{x} \tilde{f}(u, \mathbf{x}) \mu(d \mathbf{x})\right\| \\
= & o(1) \text { as } n \rightarrow \infty .
\end{aligned}
$$


Next consider (i) of (A.3). Since for any $1 \leq j \leq q, \mathcal{C}_{j n}=\cup_{k \in \mathcal{J}_{j}} \mathcal{C}_{k n}^{*}$ with the $\mathcal{C}_{k n}^{*}$ 's being disjoint, it follows that $\check{\xi}_{j n}(\mathbf{b}, u)$ is a continuous function (average) of the corresponding process based on the disjoint basic concliques $\mathcal{C}_{k n}^{*}, 1 \leq k \leq q^{*}$. Consequently, for notational simplicity, we may (without loss of generality) suppose that $\mathcal{J}_{j}=\{j\}$ for each $1 \leq j \leq q$. By using Condition (C.4), the Cramer-Wold device and the standard "big-block little block" argument for strongly mixing processes (cf. [2]), one can show that for any $\mathbf{b}_{1}, \ldots, \mathbf{b}_{k} \in \mathbb{R}^{p}, u_{1}, \ldots, u_{k} \in[0,1]$ and $1 \leq k<\infty$,

$$
\left(\check{\xi}_{n}\left(\mathbf{b}_{1}, u_{1}\right), \ldots, \check{\xi}_{n}\left(\mathbf{b}_{k}, u_{k}\right), \mathbf{z}_{n}\right) \stackrel{d}{\rightarrow}\left(\mathbf{W}\left(u_{1}\right), \ldots, \mathbf{W}\left(u_{k}\right), \mathbf{z}\right)
$$

Hence, in view of Theorem 1.5.4 of [3], it remains to show that $\left\{\check{\xi}_{n}\right\}$ is asymptotically tight. By Theorems 1.5.7 and 1.6.1 of [3], this holds provided, for any $\epsilon>0, \eta>0$, there exists a $\delta>0$ such that

$$
\limsup _{n \rightarrow \infty} P\left(\sup _{\rho_{M}(\mathbf{s}, \mathbf{t})<\delta}\left|\check{\xi}_{j n}(\mathbf{s})-\check{\xi}_{j n}(\mathbf{t})\right|>\epsilon\right)<\eta
$$

for each $1 \leq j \leq q$ and $M>0$, where $\rho(\mathbf{s}, \mathbf{t})=\left\|\mathbf{b}_{1}-\mathbf{b}_{2}\right\|^{\gamma}+\left|u_{1}-u_{2}\right|^{\gamma}$ for $\mathbf{s}=\left(\mathbf{b}_{1}, u_{1}\right), \mathbf{t}=\left(\mathbf{b}_{2}, u_{2}\right) \in \mathbb{R}^{p} \times[0,1], \gamma=1 / b$, and $\rho_{M}$ is the restriction of $\rho$ to $[-M, M]^{p} \times[0,1]$. Here, $b$ is twice the Hölder conjugate of $a$ from Condition (C.4)(iii), defined by

$$
\frac{1}{a}+\frac{2}{b}=1
$$

For notational simplicity, we shall write $\rho$ for $\rho_{M}$. We prove (A.6) in two steps. In the first step, we show that the parenthetical supremum in (A.6) differs from the supremum over $\mathbf{s}$, t's restricted to a suitable finite grid by a negligible quantity and, in the second, obtain a bound on the supremum over the finite grid using a moment bound. Let $\left\{a_{1 n}\right\}$ be a sequence of positive real numbers satisfying $a_{1 n}=o(1)$ as $n \rightarrow \infty$, where an exact convergence rate for $a_{1 n}$ will be specified later. For $\mathbf{s} \in \mathbb{Z}^{d}$ and $\theta_{0}+\mathbf{x} \in \Theta$, let $\mathbf{U}_{\theta_{0}+\mathbf{x}}^{(1)}(\mathbf{s})=(1-$ $A(\mathbf{s})) \cdot F_{\theta_{0}+\mathbf{x}}^{(1)}(Y(\mathbf{s}) \mid \mathcal{Y}(\mathcal{N}(\mathbf{s})))+A(\mathbf{s}) \cdot F_{\theta_{0}+\mathbf{x}}^{(1)-}(Y(\mathbf{s}) \mid \mathcal{Y}(\mathcal{N}(\mathbf{s})))$, where $F_{\theta_{0}}^{(1)}, F_{\theta_{0}}^{(1)-}$ denote $p \times 1$ vectors of first order partial derivatives of $F_{\theta}(\cdot \mid \cdot)$ and $F_{\theta}^{-}(\cdot \cdot \cdot)$ with respect to $\theta$. Let $\Gamma_{1 n}=\left\{\mathbf{k} \in \mathbb{Z}^{p}: \mathbf{k} a_{1 n} \in[-M, M]^{p}\right\}, \Gamma_{2 n}=\{\ell \in$ $\left.\mathbb{Z}: \ell a_{2 n} \in[0,1]\right\}$ and $\Gamma_{n}=\Gamma_{1 n} \times \Gamma_{2 n}$, where $a_{2 n}=N_{n}^{-1 / 2}\left(2 \sqrt{p} c_{0}\right) a_{1 n}$, $n \geq 1$, with $c_{0}>0$ from Condition (C.2). Fix $(\mathbf{k}, \ell) \in \Gamma_{n}$. Note that for any bivariate random vector $(X, Y)^{\prime}$ and any $x \in \mathbb{R}, \epsilon \in(0, \infty)$,

$$
\begin{aligned}
& \mathbb{I}(X+Y \leq x) \leq \mathbb{I}(X \leq x+\epsilon)+\mathbb{I}(|Y|>\epsilon) \\
& \mathbb{I}(X+Y \leq x) \geq \mathbb{I}(X \leq x-\epsilon)-\mathbb{I}(|Y|>\epsilon) .
\end{aligned}
$$


Hence, using (A.7) and Condition (C.2) with $\mathbf{b}_{1} \equiv \mathbf{k} a_{1 n} \equiv \mathbf{b}_{1}(\mathbf{k})$, it holds for large $n$ that, for any $\mathbf{b} \in\left(\mathbf{k}+[0,1]^{p}\right) a_{1 n}$ and $u \in[0,1]$,

$$
\begin{aligned}
& \left|\check{\xi}_{j n}(\mathbf{b}, u)-\check{\xi}_{j n}\left(\mathbf{b}_{1}, u\right)\right| \\
\leq & \frac{N_{n}^{1 / 2}}{\left|\mathcal{C}_{j n}\right|} \sum_{\mathbf{s} \in \mathcal{C}_{j n}} \mathbb{I}\left(\left|\tilde{U}\left(\mathbf{s}, \mathbf{b} N_{n}^{-1 / 2}\right)-\tilde{U}\left(\mathbf{s}, \mathbf{b}_{1} N_{n}^{-1 / 2}\right)\right|>a_{2 n}\right)+N_{n}^{1 / 2} \mid H_{n}(\mathbf{b}, u) \\
& -H_{n}\left(\mathbf{b}_{1}, u\right) \mid+\frac{N_{n}^{1 / 2}}{\left|\mathcal{C}_{j n}\right|} \sum_{\mathbf{s} \in \mathcal{C}_{j n}} \mathbb{I}\left(\tilde{U}\left(\mathbf{s}, \mathbf{b}_{1} N_{n}^{-1 / 2}\right) \in\left[u-a_{2 n}, u+a_{2 n}\right]\right) \\
\leq & \frac{N_{n}^{1 / 2}}{\left|\mathcal{C}_{j n}\right|} \sum_{\mathbf{s} \in \mathcal{C}_{j n}} \tilde{L}_{n}(\mathbf{s}, \mathbf{k})+\frac{N_{n}^{1 / 2}}{\left|\mathcal{C}_{j n}\right|} \sum_{\mathbf{s} \in \mathcal{C}_{j n}} L_{n}\left(\mathbf{s}, \mathbf{k}, \ell_{u}\right)+2 c_{0} \sqrt{p} a_{1 n} \\
= & 0+\frac{N_{n}^{1 / 2}}{\left|\mathcal{C}_{j n}\right|} \sum_{\mathbf{s} \in \mathcal{C}_{j n}} L_{n}\left(\mathbf{s}, \mathbf{k}, \ell_{u}\right)+2 c_{0} \sqrt{p} a_{1 n} \equiv R_{1 n}\left(\mathbf{k}, \ell_{u}\right)+2 c_{0} \sqrt{p} a_{1 n}, \text { say }
\end{aligned}
$$

where $\tilde{L}_{n}(\mathbf{s}, \mathbf{k})=\mathbb{I}\left(N_{n}^{-1 / 2}\left\|\mathbf{b}-\mathbf{b}_{1}(\mathbf{k})\right\|\left\|\mathbf{U}_{\theta_{0}+N_{n}^{-1 / 2} \mathbf{b}_{*}}^{(1)}(\mathbf{s})\right\|>a_{2 n}\right)$ for a point $\mathbf{b}_{*}=u_{*} \mathbf{b}+\left(1-u_{*}\right) \mathbf{b}_{1}(\mathbf{k})$ for some $u_{*} \in[0,1], \ell_{u}=\left\lfloor u a_{2 n}^{-1}\right\rfloor-1$ and $L_{n}(\mathbf{s}, \mathbf{k}, \ell)=\mathbb{I}\left(\tilde{U}\left(\mathbf{s}, \mathbf{b}_{1}(\mathbf{k}) N_{n}^{-1 / 2}\right) \in\left[\ell a_{2 n},(\ell+3) a_{2 n}\right]\right)$ for an integer $\ell$. Then, by Lemma A.1, Condition (C.2) and Condition (C.4)(iii), for large $n$,

$$
\begin{aligned}
& P\left(\sup \left\{R_{1 n}\left(\mathbf{k}, \ell_{u}\right): \mathbf{k} \in \Gamma_{1 n}, u \in[0,1]\right\}>\epsilon\right) \\
\leq & P\left(\max \left\{\frac{N_{n}^{1 / 2}}{\left|\mathcal{C}_{j n}\right|} \sum_{\mathbf{s} \in \mathcal{C}_{j n}} L_{n}(\mathbf{s}, \mathbf{k}, \ell): \begin{array}{l}
\mathbf{k} \in \Gamma_{1 n}, \\
0 \leq \ell+1 \leq\left\lfloor a_{2 n}^{-1}\right\rfloor
\end{array}\right\}>\epsilon\right) \\
\leq & \sum_{\mathbf{k}, \ell} P\left(\frac{N_{n}^{1 / 2}}{\left|\mathcal{C}_{j n}\right|}\left|\sum_{\mathbf{s} \in \mathcal{C}_{j n}}\left[L_{n}(\mathbf{s}, \mathbf{k}, \ell)-E L_{n}(\mathbf{s}, \mathbf{k}, \ell)\right]\right|>\epsilon / 2\right) \\
(\mathrm{A} .8) \leq & C(d, p, M) \epsilon^{-4} a_{1 n}^{-p} a_{2 n}^{-1}\left[\lambda_{n}^{-d} \pi_{0 n}^{2 / b}+\pi_{0 n}^{4 / b}\right]
\end{aligned}
$$

where $\pi_{0 n}=\max \left\{E L_{n}(\mathbf{0}, \mathbf{k}, \ell): \mathbf{k} \in \Gamma_{1 n}, 0 \leq \ell+1 \leq\left\lfloor a_{2 n}^{-1}\right\rfloor\right\} \leq C a_{2 n}$ and $\max \left\{N_{n} / \lambda_{n}^{d}, \lambda_{n}^{d} / N_{n}\right\}=O(1), \lambda_{n}^{d} /\left|\mathcal{C}_{j n}\right|=O(1)$ under the $R_{0}$ boundary condition. Similarly, for any $u \in\left[\ell a_{2 n},(\ell+1) a_{2 n}\right]$ and $\mathbf{k} \in \Gamma_{1 n}$, using the monotonicity of the function $H_{n}(\mathbf{b}, \cdot)$ and $\check{\xi}_{j n}(\mathbf{b} ; \cdot)$ and Condition (C.2), we 
get

$$
\begin{aligned}
& \left|\check{\xi}_{j n}\left(\mathbf{k} a_{1 n}, u\right)-\check{\xi}_{j n}\left(\mathbf{k} a_{1 n}, \ell a_{2 n}\right)\right| \\
\leq & \frac{N_{n}^{1 / 2}}{\left|\mathcal{C}_{j n}\right|} \sum_{\mathbf{s} \in \mathcal{C}_{j n}} \mathbb{I}\left(\tilde{U}\left(\mathbf{s}, \mathbf{b}_{1}(\mathbf{k}) N_{n}^{-1 / 2}\right) \in\left[\ell a_{2 n},(\ell+1) a_{2 n}\right]\right) \\
& +N_{n}^{1 / 2}\left|H_{n}\left(\mathbf{b}_{1}(\mathbf{k}),(\ell+1) a_{2 n}\right)-H_{n}\left(\mathbf{b}_{1}(\mathbf{k}), \ell a_{2 n}\right)\right| \\
\leq \quad & R_{2 n}(\mathbf{k}, \ell)+2 c_{0} \sqrt{p} a_{1 n}, \text { say. }
\end{aligned}
$$

As in the case of (A.8), by Lemma A.1 and Condition (C.4)(iii), for any $\epsilon>0$ and large $n$,

$$
\begin{aligned}
& P\left(\max \left\{R_{2 n}(\mathbf{k}, \ell):(\mathbf{k}, \ell) \in \Gamma_{n}\right\}>\epsilon\right) \\
\leq & C(d, p, M) \epsilon^{-4} a_{1 n}^{-p} a_{2 n}^{-1}\left[\lambda_{n}^{-d} \pi_{0 n}^{2 / b}+\pi_{0 n}^{4 / b}\right] .
\end{aligned}
$$

By choosing $a_{1 n}=\lambda_{n}^{-d\left(\frac{2}{b}-\frac{1}{2}\right) /(p+1)}$, where $2 / b=1-a^{-1}>1 / 2$ from Condition (C.4)(iii), it follows that $a_{1 n}=o(1)$ and $a_{1 n}^{-p} a_{2 n}^{-1}\left[\lambda_{n}^{-d} a_{2 n}^{2 / b}+a_{2 n}^{4 / b}\right]=o(1)$ as $n \rightarrow \infty$. Hence, by (A.8)-(A.9),

$$
\limsup _{n \rightarrow \infty} P\left(\sup _{\rho_{M}(\mathbf{s}, \mathbf{t})<C a_{1 n}^{\gamma}}\left|\check{\xi}_{j n}(\mathbf{s})-\check{\xi}_{j n}(\mathbf{t})\right|>\epsilon\right)=0 .
$$

Next, repeating the arguments leading to (A.7) and (A.8), one can show that for any $\mathbf{s}=(\mathbf{a}, u), \mathbf{t}=(\mathbf{b}, v) \in[-M, M]^{p} \times[0,1]$ with $\max \left\{\|\mathbf{a}\| N_{n}^{-1 / 2},\|\mathbf{b}\| N_{n}^{-1 / 2}, \mid u-\right.$ $v \mid\} \leq \delta_{0}$

$$
\begin{aligned}
& \left|\check{\xi}_{j n}(\mathbf{s})-\check{\xi}_{j n}(\mathbf{t})\right| \\
\leq & \left|\check{\xi}_{j n}(\mathbf{a}, u)-\check{\xi}_{j n}(\mathbf{b}, u)\right|+\left|\check{\xi}_{j n}(\mathbf{b}, u)-\check{\xi}_{j n}(\mathbf{b}, v)\right| \\
\leq & \frac{N_{n}^{1 / 2}}{\left|\mathcal{C}_{j n}\right|} \sum_{\mathbf{s} \in \mathcal{C}_{j n}} \mathbb{I}\left(\tilde{U}\left(\mathbf{s}, \mathbf{b} N_{n}^{-1 / 2}\right) \in\left[u-N_{n}^{-1 / 2} \delta_{1}, u+N_{n}^{-1 / 2} \delta_{1}\right]\right)+\delta_{1} \\
& +\frac{N_{n}^{1 / 2}}{\left|\mathcal{C}_{j n}\right|} \mid \sum_{\mathbf{s} \in \mathcal{C}_{j n}}\left[\mathbb{I}\left(\tilde{U}\left(\mathbf{s}, \mathbf{b} N_{n}^{-1 / 2}\right) \in[u, v]\right)-\left\{H_{n}(\mathbf{b}, v)-H_{n}(\mathbf{b}, u)\right\}\right],
\end{aligned}
$$

where $\delta_{1}=2 c_{0}\|\mathbf{a}-\mathbf{b}\|$. Let $r$ be an integer satisfying $2 r>(p+1) b$. Then, 
by Lemma A.1,

$$
\begin{aligned}
& E\left|\check{\xi}_{j n}(\mathbf{s})-\check{\xi}_{j n}(\mathbf{t})\right|^{2 r} \\
\leq & C\left[\|\mathbf{a}-\mathbf{b}\|^{2 r}+\sum_{k=1}^{r} \frac{N_{n}^{k}}{N_{n}^{r}}\left(N_{n}^{-1 / 2}\|\mathbf{a}-\mathbf{b}\|\right)^{\frac{2 k}{b}}+\sum_{k=1}^{r} \frac{N_{n}^{k}}{N_{n}^{r}}|u-v|^{\frac{2 k}{b}}\right] \\
(\mathrm{A} .11) \leq & C\left[\rho_{M}((\mathbf{a}, u),(\mathbf{b}, v))\right]^{2 r},
\end{aligned}
$$

Next, we apply the standard chaining argument in the proof of Theorem 2.2.4 of [3] (see pp. 98-100) with $\psi(x)=x^{2 r}, x \geq 0$ to conclude that for any $\eta>0$ and $\delta \in\left(0, \delta_{0}\right)$,

$$
\begin{aligned}
\left\|\sup _{C a_{1 n} \leq \rho_{M}(\mathbf{s}, \mathbf{t}) \leq \delta}\left|\check{\xi}_{j n}(\mathbf{s})-\check{\xi}_{j n}(\mathbf{t})\right|\right\|_{2 r} \leq C\left[\int_{C a_{1 n}}^{\eta}\left\{N\left(u, \rho_{M}\right)\right\}^{\frac{1}{2 r}} d u\right. \\
\left.+\delta\left\{N\left(\eta, \rho_{M}\right)\right\}^{\frac{1}{2 r}}\right]
\end{aligned}
$$

where $\|\cdot\|_{r}=\left[E(\cdot)^{r}\right]^{1 / r}, r \geq 1$, where $C$ is a constant not depending on $\delta, \eta$ and where $N\left(\epsilon, \rho_{M}\right)$ is the covering number of $[-M, M]^{p} \times[0,1]$ by $\rho_{M}$-balls of radius $\epsilon \in(0, \infty)$. Note that $N\left(\epsilon, \rho_{M}\right) \leq C(M, p) \epsilon^{-(p+1) / \gamma}$. Hence, setting $\eta=\delta^{1 / 2}$, the right side of the above inequality can be bounded above by

$$
C\left[\eta^{1-\frac{p+1}{2 r \gamma}}+\delta \eta^{-\frac{p+1}{2 r \gamma}}\right] \leq C \delta^{\frac{2 r-(p+1) b}{4 r}} \downarrow 0 \text { as } \delta \downarrow 0,
$$

by the condition on $r$. By (A.10) and (A.12), (A.6) follows. Hence, $\left\{\check{\xi}_{j n}\right\}_{n \geq 1}$ is asymptotically tight and in view of (A.5), (i) of (A.3) follows. This completes the proof of Theorem 4.4.

\section{APPENDIX B: PROOFS OF ADDITIONAL TECHNICAL LEMMAS}

B.1. Proof of Lemma A.1. For any integer $m \geq 2$, consider the $m$ th order cumulant, say $\chi_{m}$, of $\sum_{\mathbf{s} \in \mathcal{S}_{N_{n}}}\left[\varepsilon_{n}(\mathbf{s})-\pi_{n}(\mathbf{s})\right]$. Note that $\mid E \prod_{\mathbf{s} \in J}\left[\varepsilon_{n}(\mathbf{s})-\right.$ $\left.\pi_{n}(\mathbf{s})\right] \mid \leq C(|J|) \max \left\{\pi_{n}(\mathbf{s}): \mathbf{s} \in J\right\}$ for any finite set $J$. Hence, using the multilinearity of cumulants and the mixing condition, we may bound 


$$
\begin{aligned}
\left|\chi_{m}\right| \leq \sum_{i_{1}, \ldots, i_{m}=1}^{N_{n}}\left|\operatorname{cu}\left(\varepsilon_{n}\left(\mathbf{s}_{i_{1}}\right), \ldots, \varepsilon_{n}\left(\mathbf{s}_{i_{m}}\right)\right)\right| \text { by } & \\
& \sum_{j=0}^{N_{n}-1} \sum_{j}\left|\operatorname{cu}\left(\varepsilon_{n}\left(\mathbf{s}_{i_{1}}\right), \ldots, \varepsilon_{n}\left(\mathbf{s}_{i_{m}}\right)\right)\right| \\
\leq & \sum_{j=0}^{N_{n}-1} C(m, d) N_{n}(j+1)^{d(m-1)} \alpha(j, m-1)^{1 / a} \pi_{0 n}^{2 / b} \\
\leq & C N_{n}\left[1+\sum_{j=1}^{\infty} j^{d(m-1)} \alpha(j, m-1)^{1 / a}\right] \pi_{0 n}^{2 / b},
\end{aligned}
$$

where above $\mathrm{cu}(\cdot)$ denotes cumulant and $\sum_{j}$ denotes summation over all $1 \leq i_{1}, \ldots, i_{m} \leq N_{n}$ with maximal $\ell_{1}$-norm gap $j$. Now using the identity expressing moments in terms of cumulants, the result follows.

B.2. Proof of Lemma A.2. Let $\left\{a_{3 n}\right\}_{n \geq 1}$ be a sequence of positive real numbers such that $a_{3 n} \rightarrow 0$ as $n \rightarrow \infty$. Also, let

$$
\begin{aligned}
H_{1 n}(\mathbf{b}, u) & =P\left(U(\mathbf{0})+N_{n}^{-1 / 2} \mathbf{b}^{\prime} \mathbf{U}^{(1)}(\mathbf{0}) \leq u\right), \\
g(\mathbf{b}, u) & =\mathbf{b}^{\prime} \int \mathbf{x} \tilde{f}(u, \mathbf{x}) \mu(d \mathbf{x}),
\end{aligned}
$$

$u \in[0,1], \mathbf{b} \in \mathbb{R}^{p}$. Define $\mathbb{C}_{M} \equiv\left\{(\mathbf{b}, u): \mathbf{b} \in \mathbb{R}^{p},\|\mathbf{b}\| \leq M, u \in[0,1]\right\}$, $R_{3 n}(\mathbf{b}) \equiv \tilde{U}\left(\mathbf{0}, N_{n}^{-1 / 2} \mathbf{b}\right)-\left[U(\mathbf{0})+N_{n}^{-1 / 2} \mathbf{b}^{\prime} \mathbf{U}^{(1)}(\mathbf{0})\right], R_{4 n} \equiv \sup _{(\mathbf{b}, u) \in \mathbb{C}_{M}} \max \left\{\mid H_{1 n}(\mathbf{b}, u \pm\right.$ $\left.\left.a_{3 n}\right)-\left[u+N_{n}^{-1 / 2} g(\mathbf{b}, u)\right] \mid\right\}$ and $R_{5 n} \equiv \sup \left\{P\left(\left|R_{3 n}(\mathbf{b})\right|>a_{3 n}\right):\|\mathbf{b}\| \leq M\right\}$.

Then, using the monotonicity of $H_{n}(\mathbf{b}, u)$ in $u$, we have

$$
N_{n}^{-1 / 2} \Delta_{2 n}(M) \leq R_{4 n}+R_{5 n},
$$


and it suffices to show $R_{4 n}+R_{5 n}=o\left(N_{n}^{-1 / 2}\right)$. Note that $u=P(U(\mathbf{0}) \leq$ $u)=\int_{\mathbb{R}^{p}} \int_{-\infty}^{u} \tilde{f}(t, \mathbf{x}) d t \mu(d \mathbf{x})$ for $u \in[0,1]$. Hence,

$$
\begin{aligned}
& \sup _{(\mathbf{b}, u) \in \mathbb{C}_{M}}\left|H_{1 n}(\mathbf{b}, u)-\left[u+N_{n}^{-1 / 2} g(\mathbf{b}, u)\right]\right| \\
= & \sup _{(\mathbf{b}, u) \in \mathbb{C}_{M}}\left|\int_{\mathbb{R}^{p}} \int_{-\infty}^{u-N_{n}^{-1 / 2} \mathbf{b}^{\prime} \mathbf{x}} \tilde{f}(t, \mathbf{x}) d t \mu(d \mathbf{x})-\left[u+N_{n}^{-1 / 2} g(\mathbf{b}, u)\right]\right| \\
= & \sup _{(\mathbf{b}, u) \in \mathbb{C}_{M}} \mid \int_{\mathbf{x}: \mathbf{b}^{\prime} \mathbf{x}<0} \int_{u}^{u-N_{n}^{-1 / 2} \mathbf{b}^{\prime} \mathbf{x}}[\tilde{f}(t, \mathbf{x})-\tilde{f}(u, \mathbf{x})] d t \mu(d \mathbf{x}) \\
\leq & \sup _{(\mathbf{b}, u) \in \mathbb{C}_{M}} \int_{\mathbb{R}^{p}} \int_{u-N_{n}^{-1 / 2}\left|\mathbf{b}^{\prime} \mathbf{x}\right|}^{u+N_{n}^{-1 / 2}\left|\mathbf{b}^{\prime} \mathbf{x}\right|}|\tilde{f}(t, \mathbf{x})-\tilde{f}(u, \mathbf{x})| d t \mu(d \mathbf{x}) \\
\leq & \frac{2 M}{N_{n}^{1 / 2}} \int_{\|\mathbf{x}\| \leq b_{1 n}}\|\mathbf{x}\| \\
& \quad \sup _{t, u \in[0,1],|t-u| \leq M b_{1 n} / N_{n}^{1 / 2}}|\tilde{f}(t, \mathbf{x})-\tilde{f}(u, \mathbf{x})| \mu(d \mathbf{x}) \\
& +2 b_{1 n}^{-1} \sup _{u \in[0,1]} \int_{\|\mathbf{x}\|>b_{1 n}}\|\mathbf{x}\| \tilde{f}(u, \mathbf{x}) \mu(d \mathbf{x}) \\
= & o\left(N_{n}^{-1 / 2}\right)
\end{aligned}
$$

by Condition (C.3) if we choose

$$
b_{1 n}=N_{n}^{1 / 2}\left[N_{n}^{-1 / 2}+\sup _{u \in[0,1]} \int_{\|\mathbf{x}\|>N_{n}^{1 / 4}}\|\mathbf{x}\| \tilde{f}(u, \mathbf{x}) \mu(d \mathbf{x})\right]^{1 / 2}
$$

for which $N_{n}^{-1 / 2} b_{1 n} \rightarrow 0$ and $b_{1 n} \geq N_{n}^{1 / 4}$. By repeating similar arguments and using Condition (C.3), one gets $R_{4 n}=o\left(N_{n}^{-1 / 2}\right)+O\left(a_{3 n}\right)$. If we choose $a_{3 n}$ equal to

$$
\frac{1}{N_{n}^{1 / 2}}\left[N_{n}^{1 / 2} E\left\{\sup _{\left\|\theta-\theta_{0}\right\|<M N_{n}^{-1 / 2}}\left\|\mathbf{U}_{\theta}^{(1)}(\mathbf{0})-\mathbf{U}_{\theta_{0}}^{(1)}(\mathbf{0})\right\|\right\}\right]^{1 / 2}
$$


we have $a_{3 n}=o\left(N_{n}^{-1 / 2}\right)$ by Condition (C.2)(iii) so that $R_{4 n}=o\left(N_{n}^{-1 / 2}\right)$ and, also by Condition (C.2)(iii),

$$
\begin{aligned}
R_{5 n} & \leq \sup _{\|\mathbf{b}\| \leq M} P\left(\left|\mathbf{b}^{\prime} \int_{0}^{1}\left[\mathbf{U}_{\theta_{0}+u N_{n}^{-1 / 2} \mathbf{b}}^{(1)}(\mathbf{0})-\mathbf{U}_{\theta_{0}}^{(1)}(\mathbf{0})\right] d u\right|>a_{3 n} N_{n}^{1 / 2}\right) \\
& \leq P\left(M \sup _{\|\mathbf{x}\|<M N_{n}^{-1 / 2}}\left\|\mathbf{U}_{\theta_{0}+\mathbf{x}}^{(1)}(\mathbf{0})-\mathbf{U}_{\theta_{0}}^{(1)}(\mathbf{0})\right\|>a_{3 n} N_{n}^{1 / 2}\right) \\
& =o\left(N_{n}^{-1 / 2}\right) .
\end{aligned}
$$

This completes the proof of Lemma A.2.

\section{REFERENCES}

[1] Bolthausen, E. (1982). On the central limit theorem for stationary mixing random fields. Ann. Probab., 10, 1047-1050.

[2] Lahiri, S.N. (2003). Central limit theorems for weighted sums of a spatial process under a class of stochastic and fixed designs. . Sankhya Ser. A, 65, 356-388.

[3] van der Vaart, A.W. and Wellner, J.A., (1996). Weak convergence and empirical processes. Springer-Verlag, New York.

Department of Statistics

IOWA State University

AMEs, IA 5001

E-MAIL: mskaiser@iastate.edu dnordman@iastate.edu
Department of Statistics

Texas A\&M University

College Station, TX 77843

E-MAIL: snlahiri@stat.tamu.edu 